University of Nebraska - Lincoln

DigitalCommons@University of Nebraska - Lincoln

February 1988

\title{
Electronic structure and properties of sputtered Ta-Cu films
}

\author{
K.D. Aylesworth \\ University of Nebraska - Lincoln \\ Sitaram Jaswal \\ University of Nebraska, sjaswal1@unl.edu \\ M.A. Engelhardt \\ University of Nebraska - Lincoln \\ Z.R. Zhao \\ University of Nebraska - Lincoln \\ David J. Sellmyer \\ University of Nebraska-Lincoln, dsellmyer@unl.edu
}

Follow this and additional works at: https://digitalcommons.unl.edu/physicssellmyer

Part of the Physics Commons

Aylesworth, K.D.; Jaswal, Sitaram; Engelhardt, M.A.; Zhao, Z.R.; and Sellmyer, David J., "Electronic structure and properties of sputtered Ta-Cu films " (1988). David Sellmyer Publications. 133.

https://digitalcommons.unl.edu/physicssellmyer/133

This Article is brought to you for free and open access by the Research Papers in Physics and Astronomy at DigitalCommons@University of Nebraska - Lincoln. It has been accepted for inclusion in David Sellmyer Publications by an authorized administrator of DigitalCommons@University of Nebraska - Lincoln. 


\title{
Electronic structure and properties of sputtered Ta-Cu films
}

\author{
K. D. Aylesworth, S. S. Jaswal, M. A. Engelhardt, Z. R. Zhao, and D. J. Sellmyer \\ Behlen Laboratory of Physics, University of Nebraska, Lincoln, Nebraska 68588
}

(Received 13 July 1987)

\begin{abstract}
Films of $\mathrm{Ta}_{x} \mathrm{Cu}_{1-x}$ have been produced in a co-sputtering system with dc- and rf-sputtering guns and a substrate attached to a rapidly rotating, water-cooled table. Samples were produced in the composition range $0.1 \leq x \leq 0.9$. X-ray diffraction and electrical resistivity data between 4.2 and $300 \mathrm{~K}$ have been obtained. Diffraction measurements show that for $0.6 \leq x \leq 0.9$ the samples are glassy. The room-temperature resistivities follow the Mooij correlation with a zero temperature coefficient of resistivity at a resistivity of $35 \mu \Omega \mathrm{cm}$. The resistivity-versus-temperature data for $\mathrm{Ta}_{0.90} \mathrm{Cu}_{0.10}$ are compared to the extended Ziman-Faber theory and a localization theory by Kaveh and Mott. Of these two theories, the Kaveh-Mott theory best describes the experimental results. Ultraviolet and $x$-ray photoemission measurements have been made as a function of composition. The position of the $\mathrm{Cu} 3 d$ levels appears to be correlated with the amorphous or crystalline character of the structure. A linear muffin-tin-orbital calculation of the electronic structure of amorphous $\mathrm{Ta}_{0.59} \mathrm{Cu}_{0.41}$ has been performed. The calculation, based on a periodically extended amorphous cluster of 39 atoms, gave an electronic density of states in good agreement with photoemission results.
\end{abstract}

\section{INTRODUCTION}

Disordered and glassy metals are a challenge to understand since they require theories that differ markedly from those employed to describe crystalline and liquid states of matter. The Bloch theorem does not hold in these metals because there is no long-range order, and modeling these systems classically as "frozen" liquids does not work for electron transport, because quantum effects due to localization can appear. Several of the anomalous physical properties of disordered metals have resisted clear explanations for over a decade: In particular, these materials often exhibit negative temperature coefficients of resistivity that are difficult to reconcile with any of the existing transport theories. ${ }^{1-3}$ As a result of these circumstances, there has been considerable recent activity in this field. ${ }^{4-10}$

Among these disordered systems, refractory metal glasses are particularly interesting because of possible application in electronic devices. Specifically, glassy Ta$\mathrm{Cu}$ alloys have the potential for making excellent diffusion barriers because of their high crystallization temperatures and relatively low resistivities. ${ }^{11,12}$

While some technological aspects of $\mathrm{Ta}-\mathrm{Cu}$ glasses have been studied, no known data exist on the fundamental properties of this alloy system. This study was undertaken to provide information on the electronic structure and transport properties of these alloys. Presented here are comparisons of experimental resistivity data with two different theories, x-ray data on the structure of the $\mathrm{Ta}-\mathrm{Cu}$ system, and electronic structure information as derived from photoemission measurements and self-consistent band-structure calculations.

\section{EXPERIMENTAL METHODS}

The alloys were prepared with a multiple-gun sputtering system which is shown in Fig. 1. Prior to sample production calibration curves for the sputtering rate versus power were produced for each element. These curves were produced by sputtering a single element onto a substrate of known area for a known length of time and power. The substrates were weighed before and after deposition, and the thickness was determined using the bulk density of the element.

$\mathrm{Ta}_{x} \mathrm{Cu}_{1-x} \quad(0.1 \leq x \leq 0.9)$ films were produced by cosputtering targets of pure $\mathrm{Ta}$ and $\mathrm{Cu}$ onto a glass substrate attached to the water-cooled, rotating table as in Fig. 1. The Ta was rf sputtered and the $\mathrm{Cu}$ was dc sputtered. The total sputtering rates at the substrate were about $1 \AA / \mathrm{s}$, while the rotation rate of the table was 60 $\mathrm{rpm}$. This ensured that the samples were homogeneous alloys rather than layered materials. The films were about $3000 \AA$ thick.

The base pressure of the sputtering system was $2 \times 10^{-7}$ Torr. The sputtering was performed in a constant argon flow with the chamber pressure held at 5 mTorr.

The four-probe technique was used to measure the resistance of the films. In order to attach leads to the samples, it was necessary to sputter copper contacts onto the samples. The probe wires were then soldered to the contacts with low-melting-point $\left(47^{\circ} \mathrm{C}\right)$ solder. The resistance was measured while the probe was dipped slowly into a Dewar of liquid He. The resistance measurements were repeatable to within $0.005 \Omega$ over the entire temperature range.

The thickness of the films was inferred from the sputtering rates, and the length and width were determined with a traveling microscope. The estimated uncertainties in the resistivity, due mostly to the uncertainty in the thicknesses of the films, are about $15 \%$.

The compositions of some of the films, calculated from the sputtering rates, were compared with the compositions calculated semiquantitatively from Auger depth 


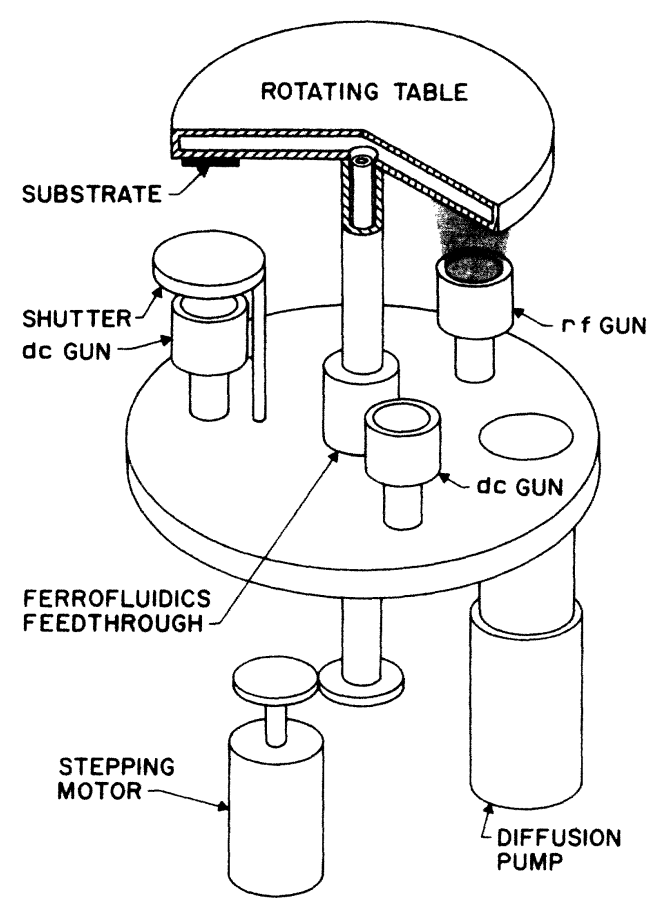

FIG. 1. A diagram of the sputtering apparatus.

profiling performed at NASA-Lewis, and were found to agree to within a few percent.

The structure of the films was studied by $\mathrm{Cu} K \alpha$ x-ray diffraction with a Phillips diffractometer. This showed that they were disordered over a large range of composition.

A Kevex Analyst 770 unit was used to perform x-ray fluorescence measurements to estimate the argon content of the films. The measurement conditions were optimized to search for argon, but the signal was less than the sensitivity of the system. This sensitivity is estimated to be considerably better than $0.1 \mathrm{wt}$. \% .

\section{RESULTS AND DISCUSSION}

\section{A. Diffraction and resistivity}

Figure 2 shows a summary of the $x$-ray data for the sputtered films. These data indicate that sputtered Ta$\mathrm{Cu}$ films are noncrystalline or glassy over the range $60-90$ at. $\%$ tantalum. In a recent study ${ }^{12}$ this range was determined to extend from 55 to 95 at. \% Ta. This corresponds well with the data for the films produced by $e$-beam evaporation. ${ }^{11}$

A plot of the temperature coefficient of resistivity versus resistivity for the entire set of samples (Fig. 3) follows the Mooij correlation, with a critical resistivity of $35 \mu \Omega \mathrm{cm}$.

Few theories of resistivity in highly disordered metals yield quantitative results that can be compared to experiment. Two theories that can be compared to experiment are the extended Ziman-Faber theory, ${ }^{13-18}$ and a localization theory of Kaveh and Mott ${ }^{19}$ that was recently ex-

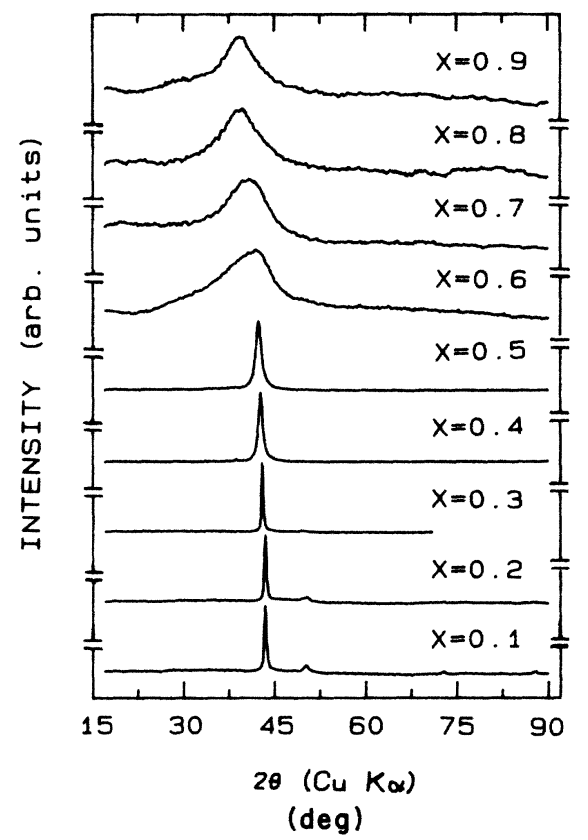

FIG. 2. A summary of the $\mathrm{x}$-ray data for sputtered $\mathrm{Ta}_{x} \mathrm{Cu}_{1-x}$ films.

tended by Tsuei. ${ }^{20}$ Other theories are discussed in Refs. 21-28.

Least-squares fits to the Ziman-Faber and Kaveh-Mott theories are shown for one glassy alloy, $\mathrm{Ta}_{0.90} \mathrm{Cu}_{0.10}$, in Figs. 4, 5, and 6.

The original Ziman-Faber theory was derived from the Boltzmann equation for liquid metals and is based on a diffraction model. In this theory the resistive structure factor and the $\mathrm{x}$-ray structure factor are assumed to be identical; the temperature dependence of this structure factor determines the temperature dependence of the resistivity. However, in disordered solids the $x$-ray and resistive structure factors are significantly different. ${ }^{13}$ The extended Ziman-Faber theory takes this difference into account.

Cote and Meise $\mathrm{l}^{13}$ obtained the following equation for resistivity based on the extended theory:

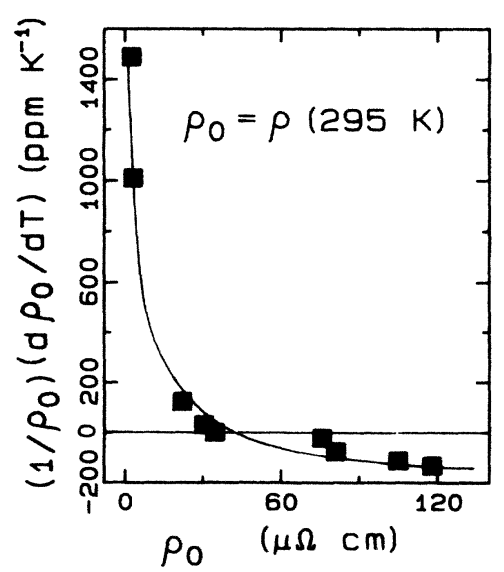

FIG. 3. A plot of temperature coefficient vs resistivity for sputtered $\mathrm{Ta}_{x} \mathrm{Cu}_{1-x}$ films. 


$$
\rho=\alpha \rho_{i}+\rho_{0} e^{-2\left[W^{*}(T)-W^{*}(0)\right]},
$$

where $\rho_{i}$ is the ideal electron-phonon resistivity and $\alpha<1$. The Debye-Waller factor is

$2 W^{*}(T)=\frac{3 \hbar^{2} K^{2}}{M k_{B} \Theta_{D}}\left(\frac{T}{\Theta_{D}}\right)^{2} \int_{0}^{\Theta_{D} / T} x\left(\frac{1}{e^{x}-1}+\frac{1}{2}\right) d x$

where $K=2 K_{F}$, and $M$ is the weighted average of the atomic masses.

The equation that was fitted to experiment is

$$
R(T)=A T+R_{0} \exp \left[-B K_{F}^{2}\left(\frac{T^{2}}{\Theta_{D}^{3}}\right) \int_{0}^{\Theta_{D} / T} \frac{x}{e^{x}-1} d x\right],
$$

where $B=12 \hbar^{2} / M k_{B}=2.13 \AA^{2} \mathrm{~K}$.

In fitting this equation to experiment the DebyeWaller factor was evaluated numerically, using an infinite series substitution. The series was truncated at each experimental point when a term in the DebyeWaller factor was smaller than $1 \times 10^{-11}$. The best least-squares fit to this equation is shown in Fig. 4. The values of the parameters obtained in the fit are $\Theta_{D}=60 \pm 10 \mathrm{~K}, A=(1 \pm 1) \times 10^{-3} \Omega \mathrm{K}^{-1}, K_{F}=0.085$ $\pm 0.005 \AA^{-1}$, and $R_{0}=29.43 \pm 0.01 \Omega$.

The values for $\Theta_{D}$ and $K_{F}$ were expected to be around $300 \mathrm{~K}$ and $1.5 \AA^{-1}$, respectively. Since the values found

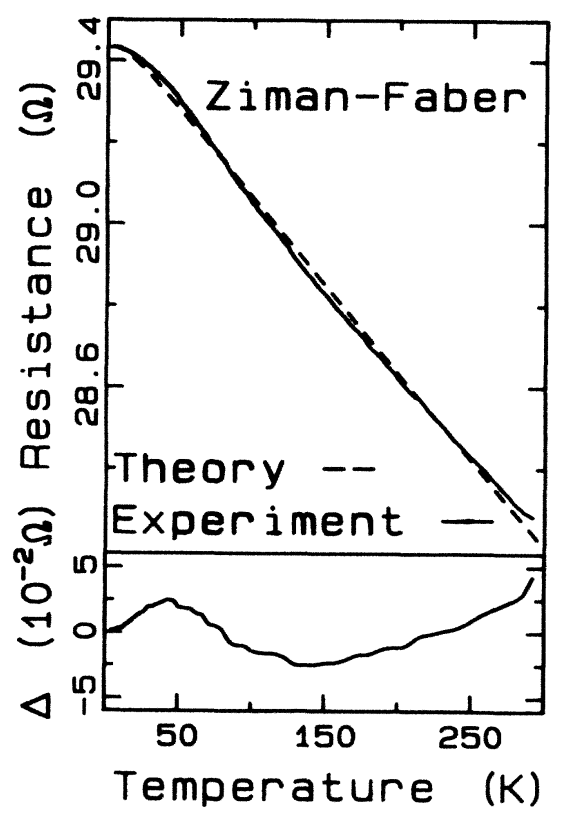

FIG. 4. A least-squares fit of the extended Ziman-Faber theory to the experimental data for $\mathrm{Ta}_{0.90} \mathrm{Cu}_{0.10} . \Delta$ is the difference between the experimental and theoretical curves. from the fit to experiment are unrealistic, it would seem that the extended Ziman-Faber theory is not likely to be adequate as a model for the resistivity of transition-metal alloys containing both $s$ and $d$ electrons.

The Kaveh-Mott theory, ${ }^{19}$ like the Ziman theory, is based upon the Boltzmann equation; however, in this case quantum corrections to the Boltzmann equation are considered. These quantum corrections are necessary because the elastic mean free path for the electrons becomes so small that the electrons are weakly localized.

Kaveh and Mott found that the corrected conductivity, assuming $\lambda_{e}<<\lambda_{i}$, is given by

$$
\begin{aligned}
\sigma(T)= & \sigma_{B}(0)\left(1-\frac{3}{\left(K_{F} \lambda_{e}\right)^{2}}\right) \\
& +\frac{e^{2}}{\pi^{2} \hbar}\left(\frac{1}{\left[\frac{1}{3} \lambda_{e} \lambda_{i}(T)\right]^{1 / 2}}-\frac{\left(K_{F} \lambda_{e}\right)^{2}}{3 \lambda_{i}(T)}\right),
\end{aligned}
$$

or

$$
\sigma(T)=A^{\prime}+B^{\prime}\left(\frac{1}{\lambda_{i}(T)}\right)^{1 / 2}-C^{\prime}\left(\frac{1}{\lambda_{i}(T)}\right)
$$

where $\lambda_{i}(T)$ is the inelastic mean free path, $\lambda_{e}$ is the elastic mean free path (assumed to be independent of temperature), and $\sigma_{B}(0)$ is the Boltzmann conductivity at $T=0 \mathrm{~K}$.

Converting this to resistance gives

$$
R(T)=\frac{1}{A^{\prime \prime}+B^{\prime \prime}\left[1 / \lambda_{i}(T)\right]^{1 / 2}-C^{\prime \prime}\left[1 / \lambda_{i}(T)\right]} .
$$

In order to fit this theory to experiment the precise temperature dependence of $\lambda_{i}$ must be known or assumed. According to this theory $1 / \lambda_{i}$ varies as $T^{p}$ $(2 \leq p \leq 5)$ as $T \rightarrow 0$ and $T^{1}$ at high temperatures. The Grüneisen function, ${ }^{29}$

$$
G_{p} \propto\left(\frac{T}{\Theta_{D}}\right)^{p} \int_{0}^{\Theta_{D} / T} \frac{x^{p} e^{x}}{\left(e^{x}-1\right)^{2}} d x
$$

fits this behavior and is assumed to determine the electron-phonon component of $\lambda_{i}$.

The experimental data are consistent with the assumption of a phonon moderated mechanism for the resistivity since the temperature coefficient of resistivity$\alpha=\rho^{-1} d \rho / d T$ - goes to zero as the temperature goes to zero.

To determine the value for $p$, the low temperature behavior of the resistivity must be examined. As $T \rightarrow 0$, Eq. (6) can be expanded in powers of $x=\left(T / \Theta_{D}\right)^{1 / 2}$ as follows: 


$$
\begin{aligned}
R(x)= & \frac{1}{A}+\left(-\frac{B p x^{p-1}-C 2 p x^{2 p-1}}{\left(A+B x^{p}-C x^{2 p}\right)^{2}}\right]_{x=0}^{x} \\
& +\frac{1}{2}\left[\frac{2\left(B p x^{p-1}-C 2 p x^{2 p-1}\right)^{2}}{\left(A+B x^{p}-C x^{2 p}\right)^{3}}-\frac{B p(p-1) x^{p-2}-C 2 p(2 p-1) x^{2 p-2}}{\left(A+B x^{p}-C x^{2 p}\right)^{2}}\right]_{x=0} x^{2} \\
& +\frac{1}{6}\left[\ldots-\frac{B p(p-1)(p-2) x^{p-3}}{\left(A+B x^{p}-C x^{2 p}\right)^{2}}+\frac{C 2 p(2 p-1)(2 p-2) x^{2 p-3}}{\left(A+B x^{p}-C x^{2 p}\right)^{2}}\right]_{x=0} x^{3}+\cdots
\end{aligned}
$$

Assuming $p=3$, the expansion above implies that as $T \rightarrow 0$

$R(x) \rightarrow \frac{1}{A}-\frac{B}{A^{2}} x^{3}=\frac{1}{A}-\frac{B}{A^{2}}\left(\frac{T}{\Theta_{D}}\right)^{3 / 2}=a-b T^{3 / 2}$

where $a=1 / A ; b=B / A^{2} \Theta_{D}^{3 / 2}$. This prediction is consistent with the Kaveh-Mott theory for a three dimensional conductor. ${ }^{19,20}$

Similarly, if $p$ is assumed to equal $4, R(T) \rightarrow a-b T^{2}$ as $T \rightarrow 0$. Unfortunately, the resolution of our data is not adequate to distinguish between these two temperature dependences.

Least-squares fits to Eq. (6) with $1 / \lambda_{i}$ proportional to $G_{3}$ and $G_{4}$ are shown in Figs. 5 and 6 . Again, the integrals were performed numerically. Table I shows the results of the least-squares fits to the Kaveh-Mott theory. The last column is the sum of the squares of the differences between experiment and theory $\left(S^{2}\right)$. These results indicate that the fit between experiment and theory is slightly better for $p=3$. This is consistent with the $R(T)=a-b T^{3 / 2}$ behavior as $T \rightarrow 0$ that was predicted for a three-dimensional conductor by Kaveh and Mott.

From the fit it can be seen that the temperature dependence is dominated by the $\left(\frac{1}{3} \lambda_{e} \lambda_{i}\right)^{-1 / 2}$ term in Eq. (4). This is reasonable since estimates of $\lambda_{i}$ and $\lambda_{e}$ for these materials are $\sim 10^{3} \AA$ and $\sim 10 \AA$, respectively.

Based upon the above results, this theory looks promising; however, in order to further determine the validity of this theory the electron-electron contribution to $\lambda_{i}$ and the temperature dependence of $\lambda_{e}$ must be included in the analysis.

\section{B. Photoemission and electronic structure}

Since no experimental atomic structure data on the $\mathrm{Ta}-\mathrm{Cu}$ system were available, the structure was determined by simulating the glass by randomly packing 23 $\mathrm{Ta}$ atoms and $16 \mathrm{Cu}$ atoms into a cube. The mass density of the glass was assumed to be $5 \%$ lower than the weighted average of the densities of crystalline $\mathrm{Ta}$ and $\mathrm{Cu}$. With these assumptions the side of the cube is 8.746 A.

Periodic boundary conditions were then applied, and the atoms were relaxed with the Lennard-Jones potential:

$$
V(r)=-\frac{A}{r^{6}}+\frac{B}{r^{12}}+C r+D .
$$

The parameters for the Ta-Ta and $\mathrm{Cu}-\mathrm{Cu}$ potentials were determined from the cohesive energy and lattice parameters of the respective crystals, with the truncation distances (where the potentials and their derivative vanish) set to $7 \AA$. The Ta-Cu parameters were then calculated by averaging the magnitudes and positions of the minima in the above potentials, and setting the truncation distance to $7 \AA$. The total and partial pair distributions determined from these potentials are shown in Figs. 7 and 8 , respectively. These pair distributions are typical of transition-metal glasses: A large main peak at the nearest neighbor distance is followed by smaller peaks corresponding to more distant neighbors.

The self-consistent calculations are based on the linear muffin-tin-orbital (LMTO) method in the semirelativistic approximation. ${ }^{30}$ The basic LMTO programs are described in Ref. 31. The Hedin-von Barth form ${ }^{32}$ of the local spin-density approximation to the exchangecorrelation potential with Janak parameters ${ }^{33}$ was used

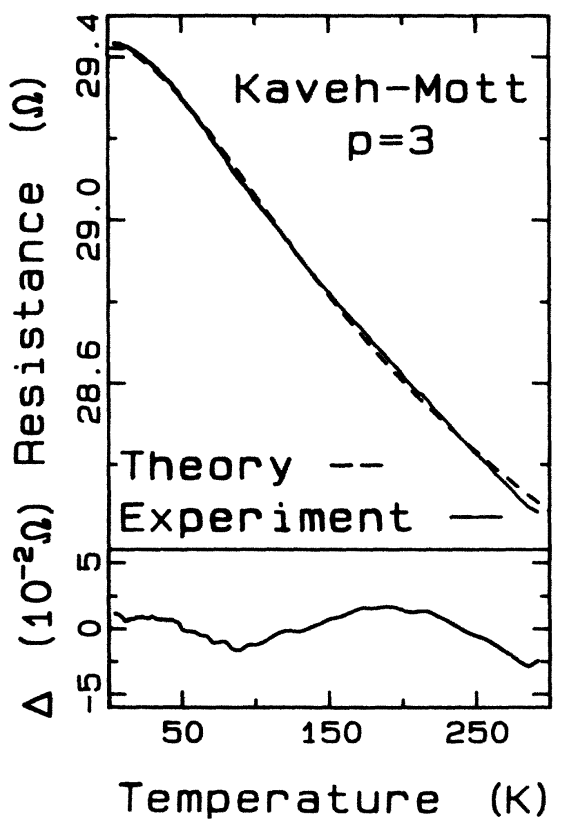

FIG. 5. A least-squares fit of the Kaveh-Mott theory $(p=3)$ to the experimental data for $\mathrm{Ta}_{0.90} \mathrm{Cu}_{0.10} . \Delta$ is the difference between the experimental and theoretical curves. 
TABLE I. The fitting parameters for the Kaveh-Mott theory with $\lambda_{i} \propto G_{p}$.

\begin{tabular}{cccccc}
\hline \hline$p$ & $a(\Omega)$ & $b\left(\Omega \mathrm{K}^{-p / 2}\right)$ & $C$ & $\Theta_{D}(\mathrm{~K})$ & $S^{2}\left(10^{-2} \Omega^{2}\right)$ \\
\hline 3 & $29.44 \pm 0.01$ & $(1.44 \pm 0.01) \times 10^{-4}$ & 0 & $770 \pm 30$ & 3.47 \\
4 & $29.43 \pm 0.01$ & $(8.62 \pm 0.01) \times 10^{-6}$ & 0 & $600 \pm 30$ & 7.87 \\
\hline \hline
\end{tabular}

in these calculations.

The Wigner-Seitz radii $\left(r_{s}\right)$ in $\mathrm{Ta}$ and $\mathrm{Cu}$ metals were used to find their values in the glass $\left[r_{s}(\mathrm{Ta})=3.18 \mathrm{a} . \mathrm{u}\right.$. and $r_{s}(\mathrm{Cu})=2.765$ a.u.].

The core states for $\mathrm{Ta}\left(1 s^{2} 2 s^{2} 2 p^{6} 3 s^{2} 3 p^{6} 3 d^{10} 4 s^{2}\right.$ $\left.4 p^{6} 4 d^{10} 4 f^{14} 5 s^{2} 5 p^{6}\right)$ and $\mathrm{Cu}\left(1 s^{2} 2 s^{2} 2 p^{6} 3 s^{2} 3 p^{6}\right)$ were frozen to be the same as the atomic states found self-consistently. The valence electrons are 5 per $\mathrm{Ta}$ and 11 per $\mathrm{Cu}$. The $s, p$, and $d$ basis functions were used for the valence states of each atom, thus the Hamiltonian and overlap matrices are $351 \times 351$.

As shown for $\mathrm{Ni}-\mathrm{Zr}$ glasses $^{34}$ simulated by a 39 -atom periodic cluster, the energy bands for this system are expected to be fairly dispersionless; hence the long-range order effects due to the periodic boundary conditions on the electronic structure must be negligible. Therefore these time-consuming self-consistent calculations are performed only at the zone center.

The calculated total and partial densities of states (DOS's) are shown in Fig. 9. The DOS is dominated by the $\mathrm{Cu} 3 d$ band centered around $3.5 \mathrm{eV}$ below the Fermi level. The density of states at the Fermi level is 0.90 states/eV atom.

X-ray photoemission spectroscopy (XPS) data for several $\mathrm{Ta}-\mathrm{Cu}$ alloys are shown in Fig. 10. It is interesting that the $\mathrm{Cu} 3 d$ peak is shifted about $1 \mathrm{eV}$ in the glassy alloys, while remaining unshifted for the crystalline alloy.

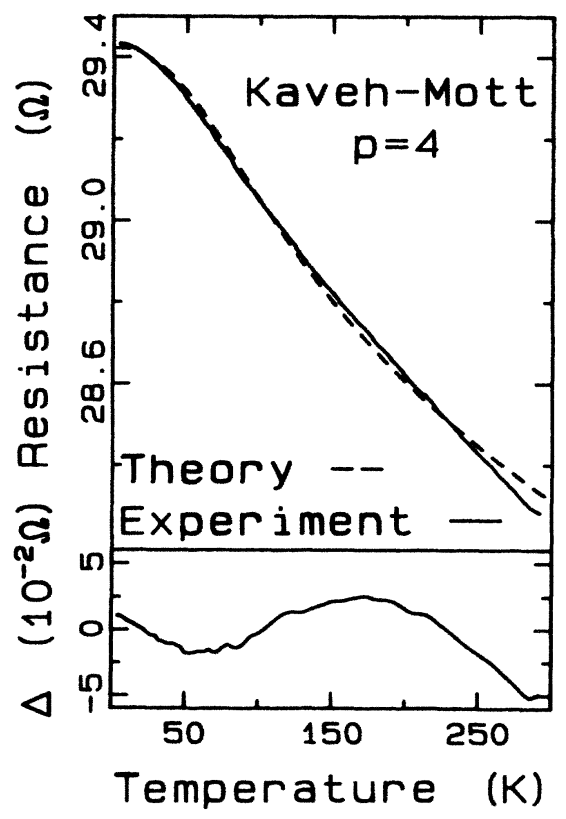

FIG. 6. A least-squares fit of the Kaveh-Mott theory $(p=4)$ to the experimental data for $\mathrm{Ta}_{0.90} \mathrm{Cu}_{0.10} . \Delta$ is the difference between the experimental and theoretical curves.
Figure 11 shows the comparison of the experimental data for $\mathrm{Ta}_{0.55} \mathrm{Cu}_{0.45}$, and LMTO calculation for $\mathrm{Ta}_{0.59} \mathrm{Cu}_{0.49}$. The slight difference in composition is not expected to significantly affect the quality of the comparison. $^{35}$ In order to compare the theory with the photoemission data, the total DOS was multiplied with the zero-temperature Fermi function, and broadened with a Gaussian of $0.3 \mathrm{eV}$ width. It is seen that the main features of the $\mathrm{Cu} 3 d$ and $\mathrm{Ta} 5 d$ states agree well in experiment and theory.

The electronic structure of $\mathrm{Ta}-\mathrm{Cu}$ is very similar to that of other early-late transition-metal combination glasses, such as $\mathrm{Zr}-\mathrm{Cu} ;{ }^{35}$ the valence band of the alloy resembles a superposition of the shifted $\mathrm{Cu} 3 d$ and Ta $5 d$ bands.

\section{SUMMARY AND CONCLUSIONS}

As expected, sputtered Ta-Cu films are glassy over a wide range of composition, and they show a negative temperature coefficient for resistivities above a critical value of $35 \mu \Omega \mathrm{cm}$. In attempting to explain this behavior, fits to two different theories were performed.

The first fit was to the extended Ziman-Faber theory. The original Ziman theory is a diffraction theory, based on the Boltzmann equation, for transport in liquid metals. The extended theory pertains to glassy metals by taking into account the difference between the resistive and $\mathrm{x}$-ray structure factors. Since the physical interpretation of the fitting parameters gives unreasonable values for the Debye temperature and Fermi momentum, this theory appears to be an inadequate model for electron transport in these glasses.

The resistivity data were also compared to a localization theory by Kaveh and Mott, and this provided a good fit with parameters that are at least of the expected order of magnitude. However, Kaiser ${ }^{36}$ has raised some objections to the assumption that the temperature

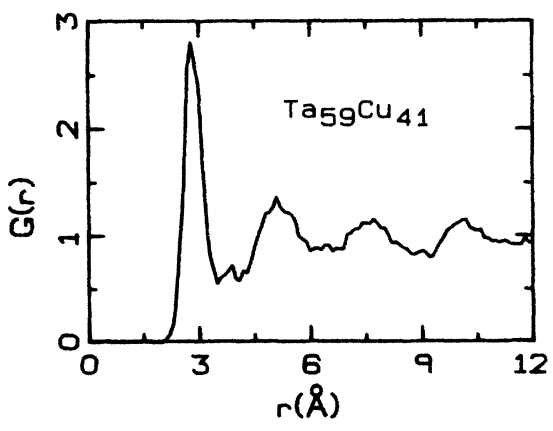

FIG. 7. Total pair distribution for $\mathrm{Ta}_{0.59} \mathrm{Cu}_{0.41}$. 


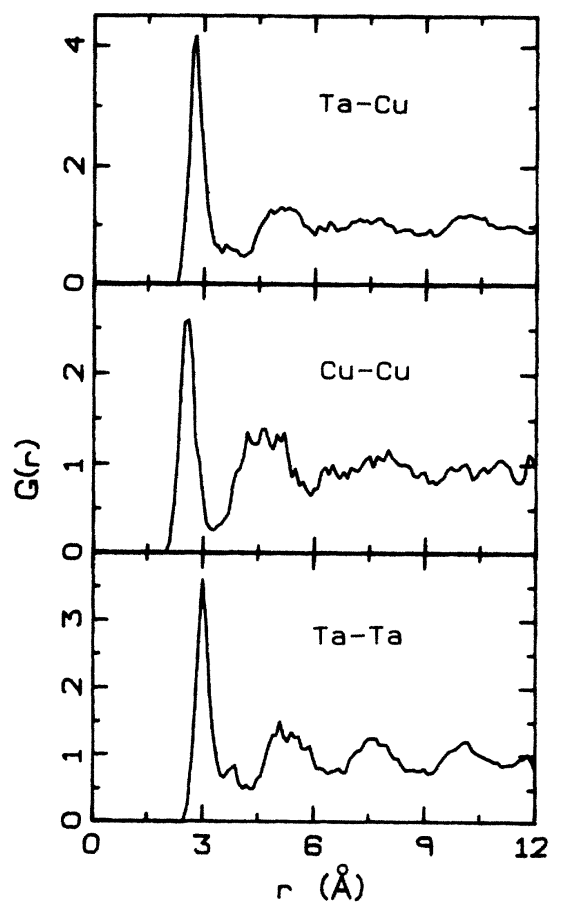

FIG. 8. Partial pair distributions for $\mathrm{Ta}-\mathrm{Cu}, \mathrm{Cu}-\mathrm{Cu}$, and Ta-Ta pairs in $\mathrm{Ta}_{0.59} \mathrm{Cu}_{0.41}$.

dependence is contained entirely in the electron-phonon contribution to the inelastic scattering term. This suggests that further theoretical work is required, particularly for glassy metals for which the critical resistivity - where $\alpha=0$-is rather small.

Finally, it was demonstrated that LMTO calculations with semiempirical pair distributions can qualitatively

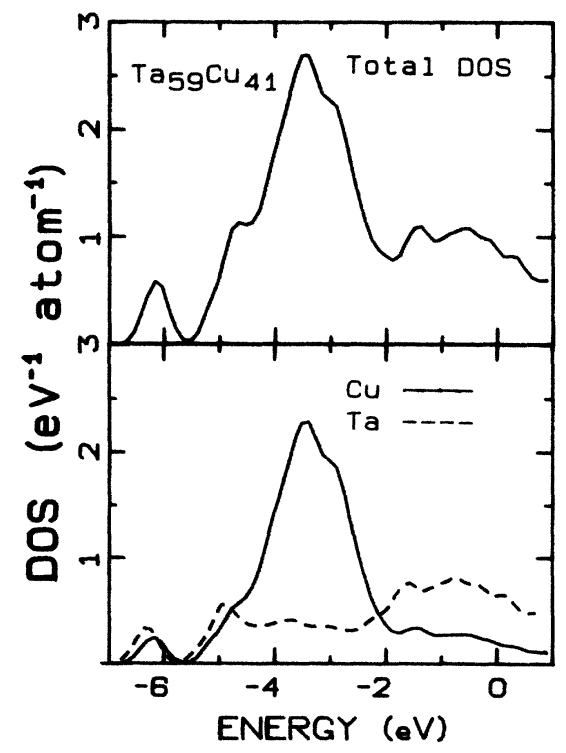

FIG. 9. Total and partial densities of states for $\mathrm{Ta}_{0.59} \mathrm{Cu}_{0.41}$.

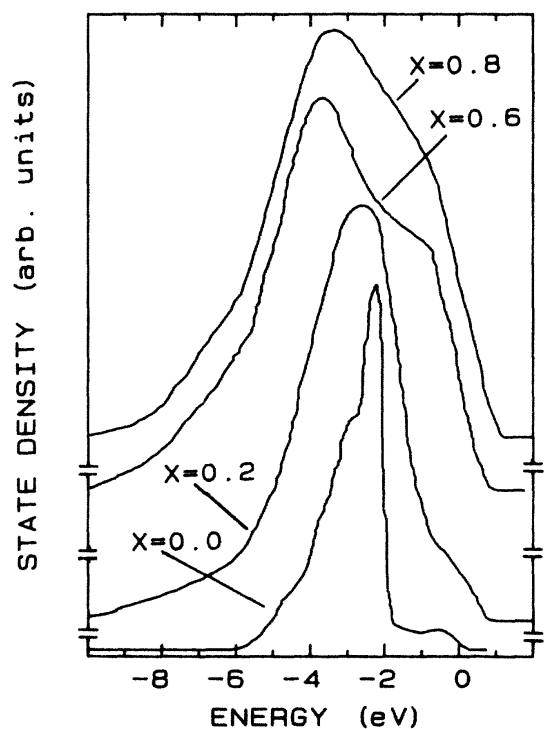

FIG. 10. XPS data for the Ta-Cu system, indicating a shift of about $1 \mathrm{eV}$ in the position of the $\mathrm{Cu} 3 d$ peak in the highly disordered alloys.
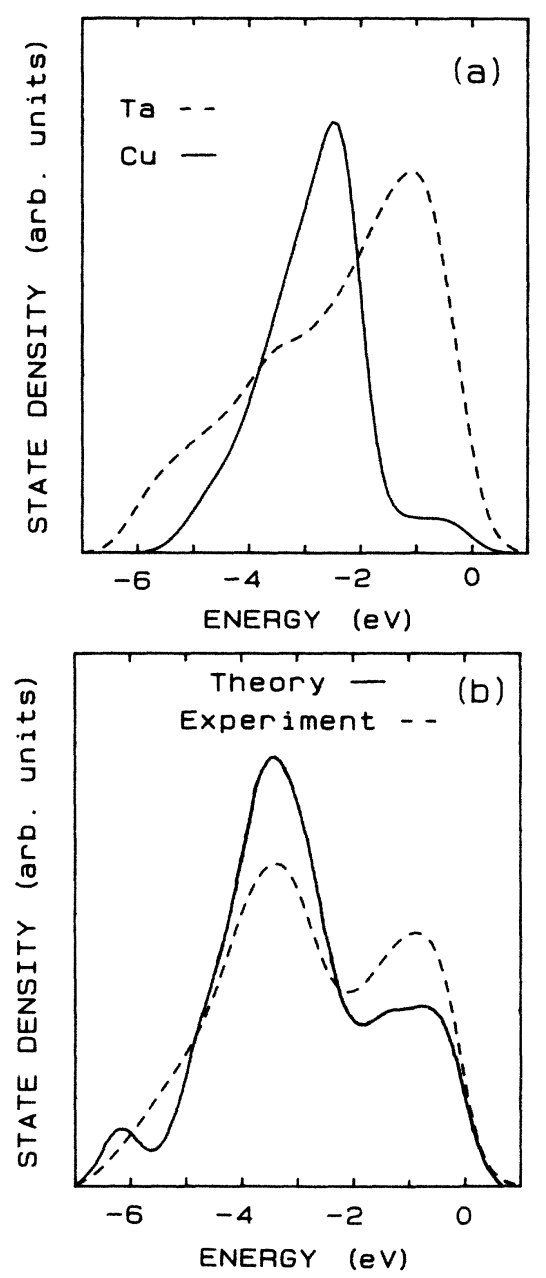

FIG. 11. (a) Photoemission data for pure $\mathrm{Ta}$ and $\mathrm{Cu}$ with $h v=21.2 \mathrm{eV}$, corrected for background. (b) Comparison of a linear muffin-tin-orbital band calculation for $\mathrm{Ta}_{0.59} \mathrm{Cu}_{0.41}$, broadened with a $0.3-\mathrm{eV}$ Gaussian, with corrected photoemission data for $\mathrm{Ta}_{0.55} \mathrm{Cu}_{0.45}(h v=21.2 \mathrm{eV})$. 
predict the band structure for these transition-metal alloys. The experimental and theoretical densities of states show that the Ta-Cu system is similar to other early-late transition-metal alloys in that the $\mathrm{Cu} 3 d$ and $\mathrm{Ta} 5 d$ bands are the main features of the band structure for the alloy. In addition, it can be seen that the shift in the $\mathrm{Cu}$ $3 d$ band predicted from theory corresponds well with the shift observed by photoemission.

\section{ACKNOWLEDGMENTS}

We would like to thank J. Oh, J. Woollam, Z. Shan, J. Pouch, and K. Xie for informative discussions and experimental assistance. For financial support we are grateful to the National Science Foundation, under Grant No. INT-8419546, and to the Dale Electronics Corporation, Norfolk, Nebraska.
${ }^{1}$ P. A. Lee and T. V. Ramakrishnan, Rev. Mod. Phys. 57, 287 (1985).

${ }^{2}$ R. Harris and J. O. Ström-Olsen, in Glassy Metals II [Top. Appl. Phys. 53, 325 (1983)].

${ }^{3}$ K. V. Rao, in Amorphous Metallic Alloys, edited by F. E. Luborsky (Butterworths, Boston, 1983).

${ }^{4}$ U. Mizutani, Y. Yamada, C. Mishima, and T. Matsuda, Solid State Commun. 62, 641 (1987).

${ }^{5}$ R. Delgado et al., Phys. Rev. B 34, 8288 (1986).

${ }^{6}$ D. G. Naugle et al., Phys. Rev. B 34, 8279 (1986).

${ }^{7}$ G. Xiao and C. L. Chien, Phys. Rev. B 34, 8430 (1986).

${ }^{8}$ S. Hershfield and V. Ambegaokar, Phys. Rev. B 34, 2147 (1986).

${ }^{9}$ J. Laakkonen and R. M. Nieminen, Phys. Rev. B 34, 567 (1986).

${ }^{10}$ S. J. Poon, K. M. Wong, and A. J. Drehman, Phys. Rev. B 31, 1668 (1985).

${ }^{11}$ F. W. Saris, L. S. Hund, M. Nastasi, J. W. Mayer, and B. Whitehead, Appl. Phys. Lett. 46, 646 (1985).

${ }^{12}$ J. E. Oh, J. A. Woollam, K. D. Aylesworth, D. J. Sellmyer, and J. J. Pouch, J. Appl. Phys. 60, 4281 (1986).

${ }^{13}$ P. J. Cote and L. V. Meisel, Top. Appl. Phys. 46, 141 (1981).

${ }^{14}$ K. D. D. Rathnayaka, A. B. Kaiser, and H. J. Trodahl, Phys. Rev. B 33, 889 (1986).

${ }^{15}$ S. R. Nagel, Phys. Rev. B 16, 1694 (1977).

${ }^{16}$ U. Mizutani and T. Yoshida, J. Phys. F 12, 2331 (1982).

${ }^{17}$ E. Babic and K. Saub, Solid State Commun. 56, 111 (1985).
${ }^{18}$ B. L. Gallagher and D. Greig, J. Phys. F 12, 1721 (1982).

${ }^{19}$ M. Kaveh and N. F. Mott, J. Phys. C 15, L707 (1982).

${ }^{20}$ C. C. Tsuei, Phys. Rev. Lett. 57, 1943 (1986).

${ }^{21} \mathrm{U}$. Mizutani, in Proceedings of the 5th International Conference on Rapidly Quenched Metals, Würzburg, edited by S. Steeb and H. Warlimont (North-Holland, New York, 1985).

${ }^{22}$ Y. Imry, Phys. Rev. Lett. 44, 469 (1980).

${ }^{23}$ D. S. McLachlan, Solid State Commun. 42, 521 (1982).

${ }^{24}$ B. L. Al'tshuler, A. G. Aronov, A. I. Larkin, and D. E. Khmel'nitskii, [Sov. Phys. - JETP 54, 411 (1981)].

${ }^{25}$ H. Raffy and R. B. Laibowitz, Phys. Rev. B 30, 5541 (1984).

${ }^{26}$ D. S. McLachlan, Phys. Rev. B 25, 2285 (1982).

${ }^{27}$ R. Cochrane, R. Harris, J. Ström-Olsen, and M. Zuckerman, Phys. Rev. Lett. 35, 676 (1975).

${ }^{28}$ C. C. Tsuei, Solid State Commun. 27, 691 (1978).

${ }^{29}$ J. M. Ziman, Electrons and Phonons (Oxford University Press, London, 1960).

${ }^{30}$ O. K. Andersen, Phys. Rev. B 12, 3060 (1975).

${ }^{31}$ H. L. Skriver, in The LMTO Method, Vol. 41 of Solid State Sciences, edited by M. Cardona, P. Fulde, and H. J. Queisser (Springer, New York, 1984).

${ }^{32}$ L. Hedin and U. von Barth, J. Phys. C 5, 1629 (1972).

${ }^{33}$ J. F. Janak, Phys. Rev. B 12, 1257 (1975).

${ }^{34}$ S. S. Jaswal, J. Non-Cryst. Solids 75, 373 (1985).

${ }^{35}$ P. Oelhafen, in Glassy Metals II [Top. Appl. Phys. 53, 283 (1983)].

${ }^{36}$ A. B. Kaiser, Phys. Rev. Lett. 58, 1384 (1987). 


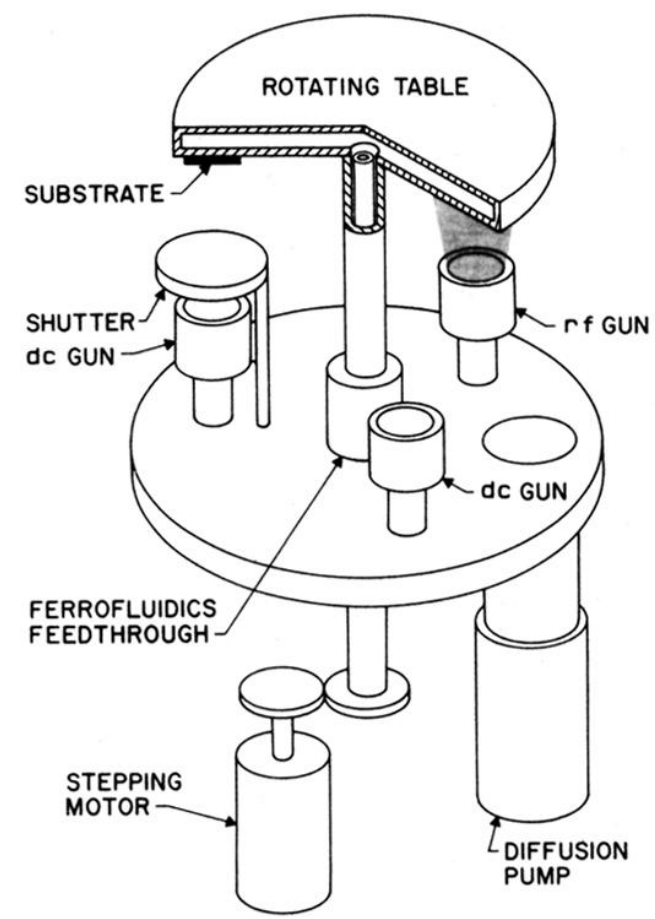

FIG. 1. A diagram of the sputtering apparatus. 